\title{
AsSociaÇÃo ENTRE DEPRESSÃo, ESTRESSE, ANSIEDADE E USO DE ÁlCOOL ENTRE SERVIDORES PÚBLICOS
}

\author{
Rejane Salomão Gavin ${ }^{1}$ \\ Emilene Reisdorfer ${ }^{2}$ \\ Edilaine Cristina da Silva Gherardi-Donato ${ }^{3}$ \\ Leonardo Naves dos Reis ${ }^{4}$ \\ Ana Carolina Guidorizzi Zanetti ${ }^{5}$
}

\begin{abstract}
Objetivo: identificar a associação de sintomatologia depressiva com variáveis sociodemográficas, exposição e dimensões do estresse ocupacional. Material e método: trata-se de estudo transversal, com 1.239 trabalhadores da categoria técnico-administrativa de uma universidade pública do interior do Estado de São Paulo. Foram avaliadas questões sociodemográficas, sintomatologia depressiva e ansiosa, uso problemático de álcool e estresse no trabalho. Resultados: os servidores públicos relataram trabalhar sob baixa demanda psicológica e alto controle sobre o trabalho executado, além de atuar com baixa exigência. Cerca um quinto dos trabalhadores apresentou sintomatologia ansiosa e/ou depressiva e 13,2\% preencheram critérios para uso problemático de álcool. Conclusão: os achados deste estudo indicam aspectos relevantes a serem enfocados por planos e estudos de intervenção, para prevenir o adoecimento mental dos trabalhadores, principalmente relacionado à sintomatologia depressiva.
\end{abstract}

Descritores: Depressão; Estresse Psicológico; Saúde do Trabalhador; Estudos Transversais.

\footnotetext{
${ }^{1}$ Mestranda, Escola de Enfermagem de Ribeirão Preto, Universidade de São Paulo, Centro Colaborador da OMS para o Desenvolvimento da Pesquisa em Enfermagem, Ribeirão Preto, SP, Brasil.

2 Pós-doutoranda, Faculty of Nursing, University of Alberta, Alberta, Canadá.

${ }^{3} \mathrm{PhD}$, Professor Associado, Escola de Enfermagem de Ribeirão Preto, Universidade de São Paulo, Centro Colaborador da OMS para o Desenvolvimento da Pesquisa em Enfermagem, Ribeirão Preto, SP, Brasil.

${ }^{4}$ Doutorando, Escola de Enfermagem de Ribeirão Preto, Universidade de São Paulo, Centro Colaborador da OMS para o Desenvolvimento da Pesquisa em Enfermagem, Ribeirão Preto, SP, Brasil.

${ }^{5}$ PhD, Professor Doutor, Escola de Enfermagem de Ribeirão Preto, Universidade de São Paulo, Centro Colaborador da OMS para o Desenvolvimento da Pesquisa em Enfermagem, Ribeirão Preto, SP, Brasil.
} 


\section{Association Between Depression, STRESS, ANXIETy AND ALCOHOL USE AMONG CIVIL SERVANTS}

Objective: To identify depressive symptoms association with sociodemographic variables, exposure and dimensions of occupational stress. Methods: This was a cross-sectional study with 1,239 workers in the technical and administrative category of a public university in the state of São Paulo. Questions were used to evaluate sociodemographic, depressive and anxiety symptoms, alcohol abuse and work stress. Results: public employees reported working under low psychological demand and had high control over the work executed, as well as working with few emergencies. About a fifth of workers presented anxious and/or depressive symptoms and $13.2 \%$ met the criteria for problematic alcohol use. Conclusion: The findings of this study indicate relevant aspects to be focused on for planning and studies for intervention, for the prevention mental illness of workers, mainly related to depressive symptoms.

Descriptors: Depression; Stress, Psychological; Occupational Health; Cross-Sectional Studies.

\section{Asociación ENTRE DEPRESIÓn, ESTRÉS, ANSIEDAD Y USO DE ALCOHOL ENTRE EMPLEADOS PÚBLICOS}

Objetivo: identificar la asociación de la sintomatología depresiva con variables sociodemográficas, exposición y dimensiones del estrés ocupacional. Material y método: se trata de un estudio transversal, con 1.239 trabajadores de la categoría técnico-administrativa de una universidad pública del interior del Estado de São Paulo. Fueron validadas cuestiones sociodemográficas, sintomatología depresiva y ansiosa, uso problemático de alcohol y estrés en el trabajo. Resultados: los empleados públicos afirmaron trabajar sobre baja demanda psicológica y alto control sobre el trabajo ejecutado, aparte de actuar con baja exigencia. Cerca de un quinto de los trabajadores presentaba sintomatología ansiosa y/o depresiva y el 13,2\% reunía las condiciones para un uso problemático de alcohol. Conclusión: los hallazgos de este estudio indican aspectos relevantes al ser abordados desde planos y estudios de intervención, para prevenir la enfermedad mental de los trabajadores, principalmente relacionado con la sintomatología depresiva.

Descriptores: Depresión; Estrés Psicológico; Salud Laboral; Estudios Transversales.

\section{Introdução}

O trabalho é uma atividade humana individual e coletiva, é uma atividade inerente ao indivíduo enquanto ser social ${ }^{(1)}$. As transformações no contexto laboral, juntamente com a globalização e os avanços tecnológicos, têm gerado alta competitividade entre as organizações. Esse fato vem provocando reestruturação das organizações no que tange aos seus processos e às 
suas atividades, causando ansiedade entre os trabalhadores, o que leva ao desgaste emocional que pode se transformar em problemas de saúde mental como depressão, estresse e ansiedade ${ }^{(2)}$.

Segundo o Ministério da Previdência Social do Brasil, os transtornos mentais e comportamentais estiveram entre as doenças com maior incidência no ano 2010, provocando um gasto total de 4,6 milhões de reais em benefícios, sendo, $84,3 \%$ previdenciários, $7,3 \%$ acidentários e 8,1 assistenciais $^{(3)}$. Esses números provocam impacto social, econômico e sobre a saúde pública no Brasil.

A depressão e o estresse são considerados problemas mentais emergentes na atual sociedade e são responsáveis por $18 \%$ das enfermidades e de absenteísmo relacionados ao contexto laboral $^{(4)}$.

Segundo a Organização Mundial da Saúde (OMS), a depressão é considerada o $5^{\circ}$ maior problema de saúde pública do mundo. Lidera as doenças mentais dos trabalhadores, pode afetar as pessoas em qualquer fase da vida, porém, a maior incidência é na idade média entre 40 e 49 anos. É mais comum no sexo feminino, com prevalência estimada de $1,9 \%$ entre os homens e de $3,2 \%$ entre as mulheres.

Entende-se depressão como um transtorno de humor grave que se caracteriza por humor deprimido ou pela perda de interesse ou prazer, presentes no período de duas ou mais semanas ${ }^{(5-6)}$. Está relacionada a consequências negativas, tanto para o trabalhador quanto para a organização em que está inserido. Causa prejuízo na qualidade de vida, interfere negativamente nas atividades cotidianas, nas habilidades funcionais no ambiente de trabalho, provoca queda da produtividade, aumento de uso de substâncias, como o uso do álcool e o tabaco e causa aumento do absenteísmo entre outras ${ }^{(7)}$.

A relação da depressão com estresse vem sendo estudada com mais ênfase, principalmente devido à importância dos problemas de saúde e de trabalho na vida do indivíduo; assim, baixos níveis de segurança, de autoridade e de poder de decisão laborais encontram-se associados ao aumento da depressão $0^{(8)}$.

O estresse é um termo que vem sendo utilizado frequentemente na atualidade para definir situações em que os indivíduos emitem respostas adaptativas a estímulos internos ou externos como fator expressivo, para o desenvolvimento ou não de um quadro patológico ${ }^{(9)}$.

Um dos referenciais teóricos mais utilizados atualmente é o Job Strain Model (JSM) ou Modelo Demanda-Controle. Conforme o JSM, situação de maior desgaste ao trabalhador e maior exposição ao estresse ocupacional, acarretando maior risco para o desenvolvimento de patologias ocupacionais, é configurada pela combinação de alto nível de demanda psicológica e baixo controle no trabalho ${ }^{(10)}$.

Assim, pode-se dizer que a depressão e o estresse são condições que podem ser consideradas recorrentes na configuração atual da sociedade, podendo ter sua patogenia, desencadeamento e evolução associados às vivências de trabalho. Discernir os fatores de risco e sintomatologias indicativos da relação entre depressão e estresse é de fundamental relevância na prevenção de agravos à saúde mental dos trabalhadores.

Neste estudo, objetivou-se identificar a associação de sintomatologia depressiva com variáveis sociodemográficas, exposição e dimensões do estresse ocupacional e uso de álcool entre servidores de uma universidade pública.

\section{Material e método}

Trata-se de estudo de corte transversal, com 1.239 trabalhadores da categoria técnico-administrativa. Os critérios de inclusão na pesquisa foram: ser funcionário da categoria técnico-administrativa e estar presente na unidade de referência no momento da coleta de dados. Optou-se por realizar o estudo por senso, englobando o todo populacional, onde a amostra ficou composta por 925 servidores participantes $(74,6 \%)$.

A coleta dos dados foi realizada por entrevistadores treinados e ocorreu por um período de 18 meses, sendo finalizada em dezembro de 2010. Aos participantes foi entregue o caderno dos instrumentos de medida, que poderia ser respondido no local de trabalho ou no domicílio, com posterior devolução à equipe.

Os instrumentos para coleta de dados deste estudo foram: Questionário de Dados Sociodemográficos (QSD), Inventário de Depressão de Beck (BDI) e Inventário de Ansiedade de Beck (BAI), Alcohol Use Disorder 
Identification Test (AUDIT) e Escala de Estresse no Trabalho - Job Stress Scale (JSS).

O Inventário de Depressão Beck (BDI) é uma escala de autorrelato, de 21 itens, cada um com quatro alternativas, submetendo a graus crescentes de depressão, com escores de 0 a 63 . O escore total é o resultado da soma dos escores individuais dos itens, que permite a classificação de níveis de intensidade de depressão. Nível mínimo ( 0 a 11 pontos), leve (12 a 19 pontos), moderado (20 a 35 pontos) e grave ( 36 a 63 pontos) $)^{(11)}$.

O Inventário de Ansiedade Beck (BAI) é constituído por 21 itens, com pontuação em escala tipo Likert de 4 pontos, que refletem níveis de gravidade crescente de cada sintoma de ansiedade (mínimo, leve, moderado e grave). A soma dos escores obtidos em cada item resulta em um escore final que varia de 0 a 63 pontos, apontando níveis: mínimo ( 0 a 10 pontos), leve (11 a 19 pontos), moderado ( 20 a 30 pontos) e grave (31 a 63 pontos) $)^{(12)}$.

Para avaliar o uso problemático de álcool foi utilizado o Alcohol Use Disorder Identification Test (AUDIT). O teste é composto por 10 questões que avaliam o uso recente de álcool, sintomas de dependência e problemas relacionados ao álcool. O escore varia de $0 \mathrm{a}$ $40 \mathrm{e}$, no presente estudo, o uso problemático de álcool foi definido pela pontuação superior a 7 pontos $^{(13)}$.

O estresse foi mensurado pela Job Stress Scale (JSS). A escala contém 17 itens distribuídos em: cinco itens pelos quais se avalia a demanda psicológica, seis itens para avaliar controle no trabalho e seis para apoio social ${ }^{(1)}$.

A partir das respostas obtidas nas dimensões demanda psicológica e controle no trabalho, as respostas foram atribuídas às quatro categorias do modelo Job Strain Model proposto por Karasek $^{(10)}$ : trabalho em baixa exigência, trabalho passivo, trabalho ativo e trabalho em alta exigência. Em seguida, os quatro tipos de trabalho foram reagrupados para fornecer a medida de exposição ao estresse ocupacional de acordo com suas três categorias, maior exposição (alta exigência), exposição intermediária (trabalhos ativo e passivo) e menor exposição ou grupo de referência (baixa exigência).

O projeto foi aprovado pelo Comitê de Ética em Pesquisa da Escola de Enfermagem de Ribeirão Preto-USP (CEP-EERP/USP), sob Protocolo n n $^{\circ} 846 / 2007$.

Foi realizada a análise descritiva dos aspectos sociodemográficos, econômicos, de trabalho, uso problemático de álcool, depressão, ansiedade e estresse ocupacional. Para a confiabilidade, foi utilizado o alfa de Cronbach. Considerou-se confiável $\alpha>0,70^{(14)}$. Os valores obtidos para JSS, BAI e BDI foram de 0,75; 0,90 e 0,85 , respectivamente, pelos quais se constatou a confiabilidade dos instrumentos para a amostra.

Para associação da sintomatologia depressiva com variáveis independentes, foi realizada regressão logística bivariada. As variáveis que apresentaram $p<0,20$, na análise bivariada foram selecionadas para compor a análise múltipla em relação à depressão, sendo submetidas à regressão logística com múltiplas variáveis. As variáveis com $\mathrm{p}>0,05$ foram retiradas para composição do modelo final, constando apenas as variáveis com $\mathrm{p}<0,05$. Utilizou-se o software estatístico STATA.

\section{Resultados}

De acordo com a análise descritiva dos dados sociodemográicos, econômicos e de trabalho, a amostra foi composta por $54,9 \%$ do sexo feminino; na faixa etária de 40 a 49 anos $(40,5 \%)$, com ensino superior completo $(51,0 \%)$, praticantes de alguma religião com $93,6 \%$, casados $(66,4 \%)$, com filhos $(69,0 \%)$, exercendo função de nível médio (52,9\%), com renda média de $\mathrm{R} \$ 4.239,50$ e com tempo de exercício do trabalho de até 10 anos $(45,9 \%)$.

Quanto aos aspectos psicossociais do trabalho, mensurados nas dimensões do JSS, os servidores públicos relataram trabalhar sob baixa demanda psicológica (53,9\%), alto controle sobre o trabalho executado $(58,1 \%)$ e alto apoio social $(42,2 \%)$. Após as combinações das demandas psicológica e controle no trabalho, as dimensões observadas entre os servidores foram: $18,7 \%$ em alta exigência, $23,0 \%$ em trabalho passivo, $27,4 \%$ em trabalho ativo e $30,9 \%$ atuavam em baixa exigência. Segundo a exposição ao estresse ocupacional, os servidores da amostra apresentaram, em sua maioria, nível médio de exposição $(50,4 \%)$, contudo, $18,7 \%$ apresentaram 
nível considerado de alta exposição ao estresse ocupacional.

Quando questionados sobre condições de saúde, 20,3\% dos trabalhadores da amostra apresentaram sintomatologia ansiosa, 21,9\% apresentou sintomatologia depressiva, $13,2 \%$ preencheram critérios para uso problemático de álcool e $6,6 \%$ relataram problemas ocorridos por uso de bebida alcoólica.

$\mathrm{Na}$ análise bivariada (Tabela 1), foram investigadas as chances de ocorrência de sintomas de depressão, de acordo com as variáveis independentes. Houve significância estatística $(p<0,05)$ para as variáveis demanda psicológica no trabalho $(\mathrm{p}=0,043)$, controle no trabalho $(\mathrm{p}<0,001)$, apoio social no trabalho $(\mathrm{p}<0,001)$, ansiedade $(\mathrm{p}<0,001)$ e problema autorreferido por uso de álcool $(\mathrm{p}<0,001)$. Essas variáveis foram incluídas no modelo de regressão logística múltipla de análise com aquelas que apresentaram $\mathrm{p}<0,20$, incluindo-se a alta exposição ao estresse ocupacional $(p=0,195)$ e o uso problemático de álcool $(\mathrm{p}=0,055)$.

Tabela 1 - Análise bivariada entre depressão e variáveis sociodemográficas, nível da função exercida, estresse ocupacional, ansiedade e uso de álcool entre servidores públicos. Ribeirão Preto, SP, Brasil, 2010

\begin{tabular}{|c|c|c|}
\hline Variável & OR (IC 95\%) & Valor de $\mathrm{p}$ \\
\hline \multicolumn{3}{|l|}{ Sexo } \\
\hline Masculino & 1 & \\
\hline Feminino & $1,21(0,86-1,72)$ & 0,264 \\
\hline \multicolumn{3}{|l|}{ Faixa etária } \\
\hline$<40$ anos & 1 & \\
\hline$\geq 40$ anos & $0,88(0,63-1,22)$ & 0,433 \\
\hline \multicolumn{3}{|l|}{ Prática religiosa } \\
\hline Não & 1 & \\
\hline Sim & $1,19(0,80-1,77)$ & 0,401 \\
\hline \multicolumn{3}{|l|}{ Filhos } \\
\hline Não & 1 & \\
\hline Sim & $1,04(0,74-1,46)$ & 0,820 \\
\hline \multicolumn{3}{|l|}{ Nível da função } \\
\hline Básico & 1 & \\
\hline Médio & $1,04(0,76-1,42)$ & 0,804 \\
\hline Superior & $0,90(0,62-1,31)$ & 0,585 \\
\hline \multicolumn{3}{|l|}{ Tempo de trabalho } \\
\hline$<15$ anos & 1 & \\
\hline$\geq 15$ anos & $1,15(0,82-1,60)$ & 0,418 \\
\hline Demanda psicológica no trabalho* & $1,04(1,00-1,08)$ & 0,043 \\
\hline Controle no trabalho* & $0,89(0,85-0,94)$ & $<0,001$ \\
\hline Apoio social no trabalho* & $0,80(0,76-0,85)$ & $<0,001$ \\
\hline \multicolumn{3}{|l|}{ Alta exposição ao estresse laboral } \\
\hline Não & 1 & \\
\hline Sim & $0,75(0,49-1,15)$ & 0,195 \\
\hline
\end{tabular}

Tabela 1 - Continuação

\begin{tabular}{lcc}
\hline Variável & OR (IC 95\%) & Valor de p \\
\hline Sintomatologia ansiosa & 1 & \\
Não & $7,77(5,49-11,00)$ & $<0,001$ \\
Sim & 1 & \\
Uso problemático de álcool & $1,52(0,99-2,33)$ & 0,055 \\
Não & & \\
Sim & 1 & \\
Problemas autorreferidos por uso de & & \\
álcool & $2,88(1,69-4,92)$ & $<0,001$ \\
Não & & \\
Sim & & \\
\hline
\end{tabular}

*Variável contínua.

$\mathrm{Na}$ análise multivariada, mostraram-se estatisticamente significativos para depressão o controle sobre o trabalho executado $(p=0,044)$, o apoio social $(p=<0,001)$, a ansiedade $(p<0,001)$ e os problemas autorreferidos por uso de álcool $(\mathrm{p}=0,001)$. As variáveis demanda psicológica, alta exposição ao estresse ocupacional e uso problemático de álcool não apresentaram significância estatística na análise multivariada, sendo retiradas do modelo final (Tabela 2).

Tabela 2 - Análise múltipla de controle no trabalho, apoio social, ansiedade e problemas autorreferidos por uso de álcool em relação à depressão entre servidores públicos. Ribeirão Preto, SP, Brasil, 2010

\begin{tabular}{lcc}
\hline Variável & OR (IC 95\%) & p \\
\hline Controle no trabalho* & $0,95(0,89-1,00)$ & 0,044 \\
Apoio social no trabalho* & $0,85(0,80-0,90)$ & $<0,001$ \\
Ansiedade & $5,97(4,14-8,60)$ & $<0,001$ \\
Problemas autorreferidos por uso de álcool & $2,76(1,51-5,04)$ & 0,001 \\
\hline *Variável contínua. & &
\end{tabular}

De acordo com os resultados da regressão logística, o controle sobre o trabalho executado atua como fator de proteção para a depressão $(\mathrm{OR}=0,95 ; \mathrm{IC} 95 \%=0,89-1,00 ; \mathrm{p}=0,044)$.

O Odds Ratio verificado na análise com múltiplas variáveis para a dimensão apoio social no trabalho $(\mathrm{OR}=0,85$; IC $95 \%=0,80-0,90$; $\mathrm{p}<0,001)$ evidenciou que essa característica laboral confere proteção para a sintomatologia depressiva.

A ansiedade apresentou-se como fator de risco para a depressão, em que os servidores com presença de sintomatologia ansiosa apresentaram 5,97 vezes mais chances de apresentar depressão em relação àqueles sem ansiedade $(\mathrm{OR}=5,97$; $\mathrm{IC}$ $95 \%=4,14-8,60 ; \mathrm{p}<0,001)$. 
Os problemas autorreferidos por uso de bebida alcoólica comportaram-se como fator de risco para sintomatologia depressiva, sendo que os servidores públicos que referiram esse tipo de problema apresentaram 2,76 vezes mais chances de depressão em relação aos que responderam negativamente a essa questão $(\mathrm{OR}=2,76$; $\mathrm{IC}$ $95 \%=1,51-5,04 ; \mathrm{p}<=0,001)$.

\section{Discussão}

No presente estudo, o controle no trabalho e o apoio social puderam ser considerados como fatores de proteção para a sintomatologia depressiva. Já a sintomatologia ansiosa e problemas autorreferidos com uso de álcool foram apontados como fatores de risco para a ocorrência do desfecho.

Outra variável considerada na literatura, a demanda psicológica no contexto laboral, foi significativa para a depressão na análise bivariada, contudo, perdeu a significância no modelo com múltiplas variáveis. Isso evidenciou que, para a amostra em estudo, a demanda apresentou-se como uma variável de confusão e que, ao ser analisada em conjunto, não tem influência sobre a sintomatologia depressiva.

O controle sobre o trabalho exercido esteve associado à depressão como fator de proteção para a sintomatologia depressiva. Vale destacar que o controle diminuiu o poder estatístico sobre a depressão quando inserido na análise múltipla, evidenciando ser uma variável com menor influência para a depressão em relação ao apoio social, à ansiedade e aos problemas autorreferidos por uso de álcool.

O alto controle sobre o trabalho executado ajuda a aumentar os níveis de satisfação no trabalho e propicia oportunidades de se envolver em tarefas desafiadoras, incrementando o repertório de habilidades do trabalhador ${ }^{(1)}$. Segundo a hipótese do efeito protetor, um nível alto de controle sobre o trabalho executado impediria que a demanda psicológica aumentasse o risco de adoecimento.

A dimensão apoio social no trabalho associada à depressão evidenciou que essa característica laboral confere proteção para a sintomatologia depressiva, sendo que as chances de apresentar depressão diminuem à medida que aumenta o escore de apoio social para a amostra em questão. $\mathrm{O}$ apoio social manteve o nível de significância estatística na análise múltipla, sendo que o Odds Ratio aumentou no modelo com múltiplas variáveis. Esse achado afirma que, ao ser analisado em conjunto, o apoio social constitui-se em uma característica psicossocial do modelo de estresse com relevada importância para o desenvolvimento de depressão, entre os trabalhadores estudados.

Em consonância com o pressuposto do modelo de estresse de Karasek, utilizado no referido estudo, o apoio social funcionou como amortecedor para a depressão, pois pode reduzir as tensões provocadas pelo estresse no ambiente de trabalho ${ }^{(15)}$.

Os mecanismos pelos quais o apoio social no ambiente laboral interfere na saúde, bem-estar e qualidade de vida são diversos. Nessa perspectiva, altos níveis de apoio social podem ser associados a melhores níveis de saúde física e psicológica, uma vez que proporcionam ao indivíduo adaptação aos efeitos deletérios de eventos estressores, diminuindo as consequências negativas sobre o organismo e propiciando bem-estar, predisposição à saúde e melhores indicadores de qualidade de $\operatorname{vida}^{(16)}$.

Considerando-se o comportamento das variáveis relacionadas ao estresse, controle, apoio social e alta exposição ao estresse, para o desfecho depressão no processo de análise, a retirada da variável alta exposição ao estresse do modelo final, por perder a significância estatística na análise múltipla, indica que, para a amostra estudada, as dimensões controle e apoio social, esse último em maior escala, são os fatores psicossociais relevantes para a depressão no contexto laboral. Esses resultados reafirmam a importância dos estudos em considerar todos os componentes psicossociais envolvidos na determinação do estresse ocupacional, e não apenas a combinação que determina o nível de exposição ao estresse.

Os problemas autorreferidos por uso de bebida alcoólica apresentaram associação significativa com a depressão. Os trabalhadores que referiram ter tido problemas decorrentes do uso de álcool apresentaram 2,76 vezes mais chances de depressão em relação aos sujeitos que não referiram essa situação. Esse resultado torna-se mais interessante pelo fato de que o uso problemático, 
identificado pela aplicação do AUDIT, não foi significativo para a depressão na análise múltipla. Pode-se hipotetizar que a questão aplicada aos participantes do estudo foi mais efetiva na identificação da situação-problema ou, ainda, que a nomenclatura do instrumento possa ter inibido a resposta dos participantes pelo fato de serem abordados em seus ambientes de trabalho.

A associação entre os problemas referidos por uso de álcool e depressão, encontrada neste estudo, está em consonância com a literatura. As pessoas que apresentam uso de álcool de risco também apresentam mais depressão em estudos nacionais e internacionais ${ }^{(17-18)}$. O uso de álcool por pessoas com depressão pode ser considerado uma estratégia de enfrentamento, sendo utilizado concomitantemente com outras medicações numa tentativa de melhor lidar com a situação ${ }^{(18)}$.

A ansiedade de nível leve a grave esteve associada à depressão entre os trabalhadores participantes do estudo. Ter ansiedade confere 5,9 vezes mais chances de apresentar depressão em relação aos sujeitos cuja mensuração de ansiedade apresentada foi mínima.

Aproximadamente metade dos pacientes diagnosticados com ansiedade também é diagnosticada com depressão e, apesar de serem transtornos clinicamente diferentes, os indivíduos podem apresentar sintomas de ansiedade e depressão conjuntamente, tais como nervosismo, irritabilidade e problemas de concentração ${ }^{(19)}$.

Em uma perspectiva biológica, verifica-se que os neurotransmissores que se relacionam com o desenvolvimento da depressão, tais como a serotonina e o Ácido Aminobutírico (GABA), também influenciam o desenvolvimento da ansiedade. Estudo realizado com 121 estudantes universitários, do interior do Estado de São Paulo, que exerciam alguma função laboral, indicou que quanto maior a ansiedade dos participantes, maiores os sintomas depressivos ${ }^{(20)}$.

\section{Conclusão}

Neste estudo, constatou-se que, para a população de trabalhadores da categoria técnico-administrativa de uma universidade pública, o controle sobre o trabalho executado e o apoio social são fatores de proteção para a sintomatologia depressiva, enquanto que a sintomatologia ansiosa e os problemas autorreferidos por uso de bebida alcoólica são fatores de risco para a depressão.

Entre os aspectos psicossociais relacionados ao estresse ocupacional, que compõem as dimensões do estresse no trabalho, a maior contribuição deste estudo é que o apoio social é o fator de proteção mais significativo para a sintomatologia depressiva mensurada nesses trabalhadores.

Os achados deste estudo indicam aspectos relevantes a serem enfocados por planos e estudos de intervenção que objetivem prevenir o adoecimento e manter a saúde mental dos trabalhadores, principalmente no que tange à sintomatologia depressiva.

\section{Referências}

1. Alves MGM, Chor D, Faerstein E, Werneck GL, Lopes CS. Estresse no trabalho e hipertensão arterial em mulheres no Estudo Pró-Saúde: Estudo Pró-Saúde (Pro-Health Study). Rev Saúde Pública. 2009;43:893-6.

2. Hazlett-Stevens H. Mindfulness-based stress reduction for comorbid anxiety and depression: case report and clinical considerations. J Nerv Ment Dis. 2012 Nov;200(11):999-1003.

3. Silva-Junior JS. Afastamento do trabalho por transtornos mentais e fatores associados: um estudo caso-controle entre trabalhadores segurados da Previdência Social. São Paulo: Universidade de São Paulo; 2012.

4. Murofuse NT, Marziale MH. Diseases of the osteomuscular system in nursing workers. Rev. Latino-Am. Enfermagem. 2005 May-Jun;13(3):364-73.

5. DSM-IV-TR. Manual diagnóstico e estatístico de transtornos mentais. 4 ed. Porto Alegre: Artmed; 2002.

6. Vera-Villarroel P, Buela-Casal G, Zych I, Cordova-Rubio N, Celis-Atenas K, Zepeda L, et al. Chilean experimental version of the StateTrait Depression Questionnaire (ST-DEP): State subscale (S-DEP). Psychol Rep. 2010 Feb;106(1):65-77.

7. Lecrubier Y. Depressive illness and disability. Eur Neuropsychopharmacol. 2000 Dec;10 Suppl 4:S439-43.

8. Schnittker J. Gene-environment correlations in the stress-depression relationship. J Health Soc Behav. 2010 Sep;51(3):229-43. 
9. Selye H. The stress of life. New York: McGraw-Hill; 1976.

10. Karasek RA, Theorell T. Healthy work-stress, productivity, and the reconstruction of working life. New York (US): Basic Books; 1990.

11. Beck AT, Steer RA, Ball R, Ranieri W. Comparison of Beck Depression Inventories -IA and -II in psychiatric outpatients. J Pers Assess. 1996 Dec;67(3):588-97.

12. Beck AT, Epstein N, Brown G, Steer RA. An inventory for measuring clinical anxiety: psychometric properties. J Consult Clin Psychol. 1988 Dec;56(6):893-7.

13. Babor TF, Higgins-Biddle JC, Saunders JB, Monteiro MG. AUDIT: The Alcohol Use Disorders Identification Test Guidelines for Use in Primary Care. 2 ed. Gèneve: Worl Health Organization; 2001.

14. Cummings SR, Stwart A, Rulley SB. Elaboração de questionários e instrumentos de coleta de dados. In: Hulley SB, Cumming SR, Browner WS, Grady DG, Hearst NB, Newman TB, editors. Delineando a pesquisa clínica Uma abordagem epidemiológica. Porto Alegre: Artmed; 2003.

15. Karasek R. The stress-disequilibrium theory: chronic disease development, low social control, and physiological de-regulation. Med Lav. 2006 Mar-Apr;97(2):258-71.

16. Fonseca ISS, Moura SB. Apoio social, saúde e trabalho: uma breve revisão. Psicol América Latina: Rev Electron Int Unión Latinoam Entidades Psicología [Internet]. 2008 [acesso 19 jan 2014];15. Disponível em: http://psicolatina. org/15/apoio.html

17. Amato TdC, Pavin T, Martins LF, Ronzani TM, Batista A. Trabalho, gênero e saúde mental: uma pesquisa quantitativa e qualitativa entre bombeiros. Cad Psicol Soc Trabalho. 2010;13:103-18.

18. Boschloo L, Vogelzangs N, van den Brink W, Smit JH, Beekman AT, Penninx BW. The role of negative emotionality and impulsivity in depressive/anxiety disorders and alcohol dependence. Psychol Med. 2012 Oct;1:1-13.

19. WHO. Conquering Depression: Some facts and figures [Internet]. WHO; 2012 [acesso em 11 ago 2012]; Disponível em: http://www. searo.who.int/en/Section 1174/Section 1199/ Section1567/Section1826_8101.htm
20. Baptista MN, Carneiro AM. Validade da escala de depressão: relação com ansiedade e stress laboral. Estud Psicol. (Campinas). 2011;28:345-52. 